\title{
Percepciones acerca de la motivación docente en personal directivo de instituciones de educación secundaria en la zona metropolitana de Medellín, 2015
}

\author{
Perceptions of High School Principals about Teacher Motivation in the Metropolitan \\ Area of Medellin, 2015
}

Hernán López-Arellano'
Instituto Tecnológico Metropolitano
Facultad de Ciencias Económicas y Administrativas
hernanlopez0429@correo.itm.edu.co
http://orcid.org/0000-0002-2575-9641
Mauricio Vélez-Salazar ${ }^{2}$
Instituto Tecnológico Metropolitano
mauriciovelez@itm.edu.co
Http://orcid.org/0000-0002-7299-5970
Jorge Ariel Franco-López

Recibido 21 de diciembre de 2015 • Corregido 10 de febrero de 2017 • Aceptado 13 de abril de 2017

Resumen: Esta es una investigación exploratoria con el propósito de medir la percepción de directores y directoras de secundaria acerca de la motivación docente, en cuatro áreas específicas: instalaciones y dotación institucional, la motivación intrínseca, motivación extrínseca e impactos sociales. En este artículo se comparan las percepciones del personal directivo de escuelas privadas y públicas, y los datos muestran una favorabilidad mayor (una mayor calificación en las categorías evaluadas) de las escuelas privadas por encima de las escuelas públicas. Los autores diseñaron y aplicaron un cuestionario de Likert de cinco escalas para determinar las percepciones acerca de la motivación en sus equipos docentes en cincuenta y un escuelas del área metropolitana de Medellín, Colombia.

Palabras clave: Percepción de la motivación; motivación docente; directores y directoras escolares; escuelas públicas y privadas.

\footnotetext{
${ }^{1}$ Magister en Ciencias de la Organización y Administrador de Empresas de la Universidad del Valle.

${ }^{2}$ Magíster en Gestión Tecnológica de la Universidad Pontificia Bolivariana e Ingeniero de Sistemas y Telecomunicaciones de la Universidad Autónoma de Manizales.

${ }^{3}$ MBA de la Universidad de Medellín, Economista y Sociólogo de la Universidad Unaula de Medellín.
} 
doi: http://dx.doi.org/10.15359/ree.21-2.5

URL: http://www.una.ac.cr/educare

CORREO: educare@una.cr

\begin{abstract}
This study is an exploratory research with the purpose of measuring perceptions High School principals have about teachers' motivation in four specific areas: facilities and institutional resources, intrinsic motivation, extrinsic motivation and social impacts. In this article, perceptions of principals working in public and private schools are compared. The authors designed and applied a five Likertscale questionnaire to determine the perceptions of principals about motivation in their teacher staffs; the tool was implemented in fifty-one schools of the Metropolitan Area of Medellín, Colombia. Data show a higher favorability (i.e., categories with a better score) in private schools than in public ones.
\end{abstract}

Keywords: Perception about motivation; teachers'motivation; school principals, public and private schools.

\title{
Introducción
}

La profesión docente se desenvuelve en un mundo altamente competitivo que exige cambios constantes en su rol. En efecto, un profesor o profesora no solo debe tener competencias científicas y técnicas, sino que debe orientar el aprendizaje de sus estudiantes al tiempo que se le exige investigar, desarrollar funciones directivas y mantenerse a la vanguardia tecnológica (González, 2003; Rodríguez, Núñez, Valle, Blas \& Rosario, 2009; Romero, 2009).

Estos desafíos han venido cambiando la forma de ejercer la profesión docente, a tal punto que es menester reflexionar constantemente sobre los procesos de enseñanzaaprendizaje (Carbonero, Martín-Antón, Román \& Reoyo, 2010), el uso de las nuevas tecnologías en la dinámica de aprendizaje (Rodríguez et al., 2009) y sus consecuencias en la relación docente-estudiante, de tal forma que se pueda mejorar continuamente la calidad de la formación en la escuela de inicios de siglo. Para hacer frente a estos retos, la escuela ha de requerir gente altamente instruida y motivada para introducir los cambios institucionales requeridos y permitir la transformación del quehacer docente (Carbonero et al., 2010) en un mundo globalizado y competitivo.

La motivación docente es un elemento fundamental para lograr satisfacción laboral y asegurar el desarrollo de procesos pedagógicos vivificantes que superen la simple satisfacción de necesidades básicas en el profesorado y permitan continuar ejerciendo su vocación con dignidad y calidad (Franco-López, López-Arellano \& Vélez-Salazar, 2015). La motivación suele determinar la conducta y el desempeño del individuo, permitiéndole alcanzar niveles mayores de eficacia en sus funciones (Ringelhan, Wollersheim, Welpe, Fiedler \& Spörrle, 2013).

Franco-López et al. (2015) encontraron suficientes evidencias para concluir que la decisión de ser docente en instituciones de educación secundaria obedecía a razones netamente individuales (factores intrínsecos) como la vocación y la posibilidad de ejercer la profesión docente en contraposición a razones externas (factores extrínsecos) tales como estímulos económicos o el reconocimiento social. Así, los autores encontraron que los grupos docentes sentían agrado por su trabajo, a pesar de considerar la actividad docente poco estimulante 
e inequitativa, dadas las diferencias salariales entre colegios públicos y privados, así como las profundas desventajas de personal docente del magisterio estatal contratado con las condiciones del decreto 1278 de 2002, frente al beneficiado con las condiciones del decreto 2277 de 1979 en el territorio colombiano.

Para 1979, el gobierno colombiano estableció en el decreto 2277 (Ministerio de Educación, 1979) las condiciones en las cuales los cuerpos educativos de entidades oficiales eran reconocidos en aspectos laborales, de acuerdo con un régimen especial en el que se primaban temas como la facilidad en el ingreso a la carrera docente, estabilidad laboral, ascensos y retiros. Se decretó un escalafón como sistema de clasificación a través de la formación académica, experiencia y méritos docentes que iban incrementando los ingresos del profesorado de manera paulatina. El decreto contempló una escala de categorías de 1 a 14 que obedecía a una clasificación de: perito o perita, experto o experta, tecnólogo o tecnóloga, normalista, institutor o instructora, normalista rural. Cada categoría representaba un nivel de ingresos que hacía atractiva la escogencia de la profesión docente en el sistema estatal escolar colombiano. Para el 2002, el gobierno colombiano expide el decreto 1278 de 2002 (MEP, 2002), en el que el régimen de privilegios del magisterio estatal fue desarticulado Este decreto tuvo como objetivo generar un estatuto de profesionalización que regulara las situaciones contractuales entre Estado y personal educativo a partir del año 2002. Argumenta que las causas de su expedición obedecieron a la aguda situación fiscal de Estado colombiano. Con ello, se eliminó el anterior escalafón establecido en el decreto del año 1979, así como sus prerrogativas. De esta manera, el ingreso a la actividad docente en el sistema estatal colombiano mantuvo el concurso de méritos para acceder a la carrera profesoral; pero añade requisitos para la conservación del cargo a través de una evaluación de desempeño anual. Cambió también la categorización profesoral a tres grados y cuatro niveles (Art. 20) y el salario se asigna de acuerdo con esta escala. Otro cambio introducido por el decreto 1278 de 2002 en materia de ascensos es la obligatoriedad de obtener una evaluación de desempeño del $80 \%$ para acceder a este derecho.

Ahora bien, se tuvo como objeto en la investigación la medición de la motivación de docentes de instituciones de educación secundaria, percibida desde los grupos directivos o administrativos académicos. La información se obtuvo en cincuenta y una instituciones de educación secundaria en el área metropolitana de Medellín durante el año 2015. En síntesis, el propósito fue medir, de manera exploratoria, y desde la óptica administrativa, las percepciones acerca de cuatro factores en los que se enmarca la profesión docente, a saber: dotación institucional, factores intrínsecos, factores extrínsecos y el impacto social de la institución. 
doi: http://dx.doi.org/10.15359/ree.21-2.5

URL: http://www.una.ac.cr/educare

CORREO: educare@una.cr

\section{Revisión bibliográfica}

La desmotivación está presente en cualquiera de los ámbitos del ser humano y puede afectar la forma de alcanzar las metas del individuo, particularmente en las áreas del conocimiento y desarrollo humano relacionadas con el oficio de la docencia (Torres, 2006). El quehacer docente ha venido transformándose debido a la ampliación de sus funciones que involucran tareas administrativas y de investigación pedagógica, que redundan en el cumplimiento de horarios estrictos, atención a estudiantes, asistencia a reuniones, elaboración de documentos, preparación de informes de gestión, entre otros (Romero, 2009). Estos desafíos exigen nuevas competencias para hacer frente a los nuevos cargos académico-administrativos, contextualizados en un cambio de paradigma doble: de uno tradicional, centrado en el personal docente, a uno centrado en el estudiantado; y el cambio de roles consecuencia de las nuevas funciones a que está expuesta la docencia, que transforma la actividad de difundir saberes hacia la creación y gestión de conocimientos a través de la investigación (Carbonero et al., 2010).

Otros factores de desmotivación identificados en la profesión docente son: el alto volumen de tareas en el limitado horario de trabajo, problemas de disciplina y convivencia de sus estudiantes, mala actitud y motivación baja hacia el aprendizaje de sus estudiantes, órdenes ministeriales que cambian el currículo, falta de apoyo de colegas de trabajo (Romero, 2009), la compensación salarial y el no reconocimiento social de la profesión (Franco-López et al., 2015), las críticas recibidas por la sociedad y la falta de apoyo de las directivas para desenvolverse con plenitud en las aulas de clase (Torres, 2006).

De otro lado, el nuevo siglo trae consigo retos como la globalización, la tecnología inalámbrica, la multiculturalidad, la competencia internacional, la internacionalización del currículo, entre otros, poniendo a los docentes en dificultosa posición ante las nuevas demandas educativas en las áreas de ciencia, tecnología e innovación, consecuencia de una reestructuración económica y social que no conoce fronteras. Ello obliga a la reinvención de la escuela para adaptarla a las nuevas demandas (González, 2003) y al profesorado a ser artífice del cambio.

\section{La motivación}

La motivación es un término derivado de la palabra latina movere, que significa movimiento (Latham, 2007) y agrupa una diversa serie de deseos, necesidades, impulsos, anhelos y fuerzas internas similares en los individuos que tiene origen en el entorno cultural. Estos impulsos influyen en la interpretación individual acerca de la realidad y afectan la manera en que se construyen las percepciones u opiniones frente al trabajo y a la forma en la que se conduce la vida personal (Davis \& Newstron, 2003). 
Efectivamente, existe una profunda relación entre motivación y la conducta mostrada por los sujetos tanto en su vida privada como en los niveles de desempeño en su trabajo (Navarro, 2008). Una persona desmotivada puede mostrarse indiferente, agresiva, depresiva y poco productiva. En contraposición, las motivadas muestran niveles de energía y de concentración mayores hacia el logro de una tarea, así como la persistencia necesaria y las habilidades y estrategias pertinentes para alcanzar sus metas (Locke \& Latham, 2002, 2013; Locke, Shaw, Saari \& Latham, 1981).

La motivación es más que una simple lista de deseos y necesidades humanas que pueden ser analizadas por un ente observador entrenado (Araque \& Rivera, 2005), en contraste, es un proceso complejo que canaliza energías e influye en la forma cómo los seres humanos persiguen sus fines o intereses y los medios utilizados para alcanzarlos. De ahí que, al estudiar la motivación, no solo se haga énfasis en determinar los motivos y los recursos utilizados para alcanzar los objetivos trazados, sino también en las habilidades requeridas para la tarea (Vroom, 1964) y las recompensas vinculadas al desempeño de esta, sean ellos incentivos tangibles mediatos (Skinner, 1981), compensaciones futuras (Vroom, 1964), gratificaciones personales derivadas del interés propio (Deci, 1980; Gagné \& Deci, 2005, 2014), motivos de índole cognitiva como dar significado a la existencia personal (Bandura, 1988, 1991; Wood \& Bandura, 1989) o el compromiso adquirido con la tarea al convertir las intenciones personales -deseo esto- en conductas dirigidas hacia el alcance de metas -haré esto- (Gollwitzer, 1999; Gollwitzer \& Sheeran, 2006).

Para la psicología científica, la motivación se relaciona con distintas elaboraciones empíricas y teóricas cuyo propósito principal es describir, explicar y predecir el comportamiento humano (Herrera \& Matos, 2009). El hecho de que las teorías acerca de las necesidades humanas busquen, con afán, entender qué hace a un individuo desempeñarse bien en una tarea (Alderfer, 1972; Herzberg, 2003; Herzberg, Mausner \& Bloch, 2010; Maslow, 1991) es ya de por sí uno de los debates clásicos en la gerencia moderna a la que se le han ido añadiendo elementos de la gerencia como el ejercicio del poder (McClelland, 1989), el liderazgo (Bennis, 1966; Kirkpatrick \& Locke, 1991; McGregor, 1971), sistemas de control y retroalimentación (Campion \& Lord, 1982), las teorías acerca de la equidad y la justicia (Adams, 1963; Thierry, 2001), entre otras. 
doi: http://dx.doi.org/10.15359/ree.21-2.5

URL: http://www.una.ac.cr/educare

CORREO: educare@una.cr

En la Figura 1 se clasifican las distintas teorías motivacionales, según Mitchell y Daniels (2003):

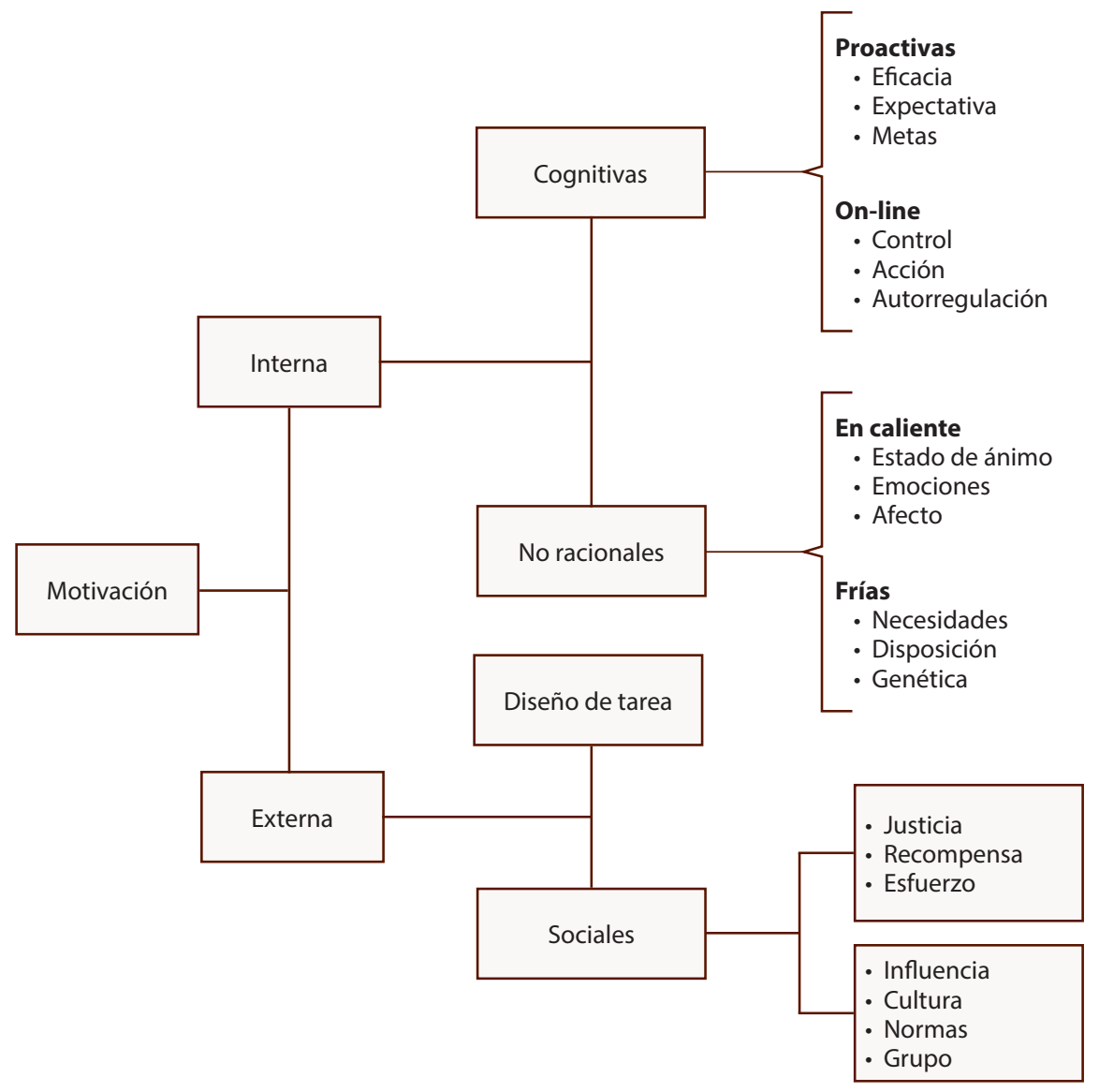

Figura 1: Revisión de las teorías de la motivación elaborada a partir de Mitchell \& Daniels, 2003.

\section{Motivación intrínseca}

Los estudios de motivación han intentado aclarar las causas de la conducta y determinar los motivos que hacen que una persona realice una acción dirigida hacia fines concretos (Araque \& Rivera, 2005; Donovan, 2002). Los motivos han sido clasificados en dos categorías: las motivaciones internas que se derivan de las necesidades, las cogniciones y emociones de los individuos; y las motivaciones externas, las cuales pretenden determinar cómo operan los estímulos o el conjunto de acontecimientos exteriores que, debido a las consecuencias sociales, ambientales y culturales, brindan energía a la conducta, como por ejemplo, los incentivos monetarios o las recompensas sociales.

Las teorías que se han enfocado en el estudio de las motivaciones internas de los individuos pueden, a su vez, ser clasificadas en dos: cognitivas y no racionales. Las cognitivas se orientan al entendimiento de los procesos mentales conscientes que operan en el interior del individuo y le 
permiten alcanzar niveles mayores de desempeño; factores tales como las metas, la auto-eficacia y las expectativas en el comportamiento del individuo (on-line) frente a determinados objetivos, o bien, qué tipo de influencia tienen el control, la acción y la autorregulación (proactivas) en la conducta. Así, estas teorías buscan entender el proceso mental que tendrá repercusiones en la conducta del ser humano antes de iniciar una tarea (Mitchell \& Daniels, 2003).

De otro lado, las teorías "no racionales" se ocupan de los procesos que ocurren durante la tarea (Mitchell \& Daniels, 2003). Las teorías no racionales buscan determinar la influencia de factores "en caliente" (hot) o en el momento en que se desarrolla la tarea, como el estado de ánimo, las emociones, el afecto. Por su parte, las teorías frías (cold) son menos maleables y ponen especial atención a factores como las necesidades, la predisposición personal y la expresión génica (Mitchell \& Daniels, 2003).

La forma en que opera la motivación intrínseca en el individuo es un factor determinante en la percepción individual acerca de la escogencia de una profesión como la docente, así como en la percepción individual de haber desarrollado las competencias necesarias para ejercerla (Deci, 1980; Gagné \& Deci, 2005).

La motivación intrínseca se encuentra relacionada con la capacidad de los seres humanos para obtener satisfacción de lo que hacen (Gagné \& Deci, 2005), en oposición a las teorías ampliamente aceptadas en las cuales incentivos externos tienen relación directa con el desempeño de las tareas. Varios estudios apuntan a que actividades desafiantes, así como la retroalimentación positiva, tienen efectos directos en las sensaciones de competencia y autonomía personales que influencian la satisfacción de las personas en su lugar de trabajo y en su vida. Justamente, un personal docente motivado se relaciona con el desarrollo de funciones con autonomía, implicación personal, iniciativa, perseverancia y compromiso de cambio hacia el mejoramiento de la calidad de la enseñanza (Lauretti, Villalobos \& González, 2007).

Existe gran cantidad de estudios que muestran la motivación intrínseca como un elemento fundamental en el desempeño de las personas en ambientes académicos, dado que involucra percepciones individuales descritas como labores emocionantes y desafiantes (Ringelhan et al., 2013), así como la existencia de una cierta cantidad de autonomía, espacio para la creatividad, variabilidad en las labores y propósitos estimulantes y envolventes (Amabile, 1997; Deci, 1980). Ringelhan et al. (2013) sugieren, incluso, que el personal académico con alto grado de motivación intrínseca, son grupos trabajadores más comprometidos, persistentes, ponen mayor énfasis en su desempeño y establecen metas más exigentes que sus colegas con un menor grado de motivación.

La motivación intrínseca está directamente relacionada con la autorregulación, es decir, con procesos psicológicos que permiten regular la conducta propia hacia la obtención de objetivos individuales (Panadero \& Alonso-Tapia, 2014), ello implica el conocimiento previo de los resultados finales y las relaciones causa-efecto del comportamiento (Bandura, 1988, 1991). La autorregulación involucra procesos a través de la modulación del pensamiento, el afecto y la conducta que capacitan a las personas para guiar sus acciones hacia el alcance de metas en el tiempo (Latham, 2007). 
doi: http://dx.doi.org/10.15359/ree.21-2.5

URL: http://www.una.ac.cr/educare

CORREO: educare@una.cr

Como lo expresa Bandura (1991), el pensamiento regula los actos humanos en su mayor parte. Así, las personas forman juicios acerca de sus competencias, anticipan las consecuencias de sus acciones, establecen metas para sí mismas y planean cursos de acción con la intención de alcanzarlas. En efecto, los individuos autorregulan sus emociones, sus cogniciones y acciones con la intención de reducir las discrepancias entre su "yo real" y su "yo ideal" (Moretti \& Higgins, 1999).

Como la autorregulación está directamente relacionada con los propósitos individuales que dan significado a la existencia, se desprende que el futuro está presente en el funcionamiento cognitivo de las personas. Así, cada quien posee una representación mental de lo que quiere llegar a ser (expectativa) y esa imagen es lo que dirige y regula su conducta, junto con otros mecanismos cognitivos como la autoevaluación, la auto-observación y la autorreacción (Bandura, 1988, 1991). En la Tabla 1 se puede observar una breve descripción de cada una de las teorías que se enfocan en el estudio de la motivación intrínseca y sus principales exponentes.

Tabla 1: Teorías de la motivación intrínseca

\begin{tabular}{|c|c|c|}
\hline Escuela & Principales exponentes & Breve descripción \\
\hline $\begin{array}{l}\text { Teoría de la evaluación } \\
\text { cognitiva o la } \\
\text { autodeterminación }\end{array}$ & $\begin{array}{l}\text { (Deci, 1972, 1980; Gagné \& } \\
\text { Deci, 2014) }\end{array}$ & $\begin{array}{l}\text { La motivación tiene su origen en el individuo, es decir, sin } \\
\text { recompensa externa aparente, excepto la obtenida por } \\
\text { desarrollar la actividad en sí misma o los sentimientos } \\
\text { que resultan de esa actividad. }\end{array}$ \\
\hline $\begin{array}{l}\text { Teoría de fijación } \\
\text { de objetivos (Goal } \\
\text { Setting Theory) }\end{array}$ & $\begin{array}{l}\text { (Locke \& Latham, 2002, } \\
\text { 2013; Locke et al., 1981) }\end{array}$ & $\begin{array}{l}\text { Se enmarca en el dominio de la psicología cognitiva y } \\
\text { se basa en la idea de que la mayoría de las conductas } \\
\text { humanas son el resultado de escoger conscientemente } \\
\text { metas e intenciones determinadas. }\end{array}$ \\
\hline Teoría del control & $\begin{array}{l}\text { (Campion \& Lord, 1982) } \\
\text { (Carver \& Scheier, 1981, } \\
\text { 1990) }\end{array}$ & $\begin{array}{l}\text { Esta teoría menciona que la motivación hacia una meta } \\
\text { inicia con la identificación de una discrepancia entre } \\
\text { lo deseado (valor de referencia) y el estado actual. En } \\
\text { consecuencia, seforma un mecanismo de autocorrección } \\
\text { (motivador) que genera una conducta (acción) en el } \\
\text { individuo para disminuir o eliminar la discrepancia. }\end{array}$ \\
\hline Teoría socio-cognitiva & $\begin{array}{l}\text { (Bandura, 1988, 1991; } \\
\text { Weinberg, Gould \& Jackson, } \\
\text { 1979; Wood \& Bandura, } \\
\text { 1989) }\end{array}$ & $\begin{array}{l}\text { Afirma que las metas y proyectos de un individuo tienen un } \\
\text { poder motivacional sobre él, de este modo, el sujeto actúa } \\
\text { en función de un propósito y todos sus sistemas se dirigen } \\
\text { a alcanzar las metas que darán significado a su vida. }\end{array}$ \\
\hline $\begin{array}{l}\text { Teorías de la } \\
\text { motivación on-line }\end{array}$ & $\begin{array}{l}\text { (Achtziger, Gollwitzer \& } \\
\text { Sheeran, 2008; Gollwitzer, } \\
\text { 1999); Gollwitzer \& Sheeran, } \\
2006\end{array}$ & $\begin{array}{l}\text { Teoría que se enfocan en determinar el proceso que } \\
\text { ocurre luego de que un propósito ha sido aceptado por un } \\
\text { individuo. Así, las personas convierten sus intenciones de } \\
\text { implementación (quiero hacer esto) en conductas hacia el } \\
\text { logro de objetivos deseados (quiero esto). }\end{array}$ \\
\hline
\end{tabular}

Nota: Elaboración propia a partir de Donovan (2002) y Mitchell \& Daniels (2003). 


\section{Motivación extrínseca}

En la bibliografía científica, se dice que una persona es motivada de dos maneras: a través de incentivos o recompensas, llamados también factores extrínsecos, así como motivaciones y aspiraciones internas conocidas también como factores intrínsecos (Donovan, 2002). En este segundo grupo se encuentran las investigaciones que hacen un mayor énfasis en las situaciones de alta incertidumbre, en las que se involucran docentes de secundaria, las cuales generan sentimientos de angustia al enfrentar la poca retroalimentación de su desempeño en relación con el proceso de aprendizaje de sus estudiantes (Emo, 2015). En efecto, la percepción docente respecto de su trabajo en el aula de clase afecta tanto su motivación como su efectividad (Day, Sammons, Stobart, Kington y Gu, 2007), al limitar la retroalimentación de parte de la gerencia hacia ellos o ellas.

Este grupo de investigaciones intenta determinar los factores externos que generan ansiedad en docentes debido a una serie de situaciones a las que se enfrentan -poca retroalimentación en el trabajo, uso de nuevas metodologías para la formación de estudiantes, hasta una incesante presión del medio académico para el cambio en los patrones de enseñanza-(Emo, 2015).

De acuerdo con Feldman (2013), los enfoques motivacionales basados en incentivos, se derivan del deseo de alcanzar recompensas externas. Así, el individuo se ve enfrentado a propiedades deseables deestímulos externos, sean estas calificaciones, dinero, afecto, comida o sexo que impulsan la conducta, como, por ejemplo, obtener una mayor calificación o una bonificación pecuniaria. Así mismo, ante la falta de estímulos internos aparentes, una persona puede experimentar el impulso de satisfacer necesidades, incluso cuando los incentivos no son evidentes.

El conjunto de teorías que se enfocan en el estudio de las motivaciones externas de los individuos también pueden ser clasificadas en dos: las que se ocupan del diseño de la tarea y las que describen la influencia social en la conducta (Mitchell \& Daniels, 2003). En el primer grupo, podemos ubicar los trabajos de Frederick Herzberg y su teoría motivación-higiene, él habla de un componente necesario en el entendimiento de la motivación humana al que llama KITA, que puede entenderse como el intento externo impuesto por la gerencia para instalar un "generador" en el personal (Herzberg, 2003).

KITA no es más que una forma de impulsar, sea física o psicológicamente, a una persona para realizar la labor encomendada. El dilema surge cuando se produce una KITA física negativa (pegarle al sujeto empleado) o una KITA psicológica negativa (agredirle verbalmente), pues se está contradiciendo la imagen de benevolencia que han expresado exponentes de la teoría administrativa como la mejor forma de hacer productivos al personal empleado. El resultado es un incesante mecanismo de violencia hacia el sujeto empleado que traerá como consecuencia su desmotivación (Herzberg, 2003).

Para Herzberg (2003), existen dos factores a considerar en el estudio sobre motivación, los cuales pueden ser encontrados en la bibliografía con cualquiera de los siguientes nombres 
doi: http://dx.doi.org/10.15359/ree.21-2.5

URL: http://www.una.ac.cr/educare

CORREO: educare@una.cr

(Murillo et al., 2007): insatisfactores-satisfactores, factores de higiene-motivadores o factores extrínsecos-intrínsecos. Herzberg (2003) encontró que los factores higiénicos o insatisfactores eran todas aquellas condiciones que rodean a la persona empleada mientras desarrolla su labor e incluye: condiciones físicas y ambientales de supervisión, el clima de relaciones entre dirección y personal, reglamentos internos, oportunidades existentes, entre otros. En otras palabras, son todas aquellas situaciones que se encuentran en el ambiente y constituyen todos los factores que tradicionalmente habían sido usados por las empresas para motivar a sus empleados y empleadas.

Encontraste,losfactores motivadores o"satisfactores"se referían a todos aquellos componentes relacionados con el contenido del cargo, las tareas y los deberes oficiales del cargo. Este tipo de factores produce efectos de complacencia duraderos en el individuo y aumentan su productividad, entre ellos podemos enumerar: el logro, el reconocimiento, el trabajo en sí mismo, la responsabilidad (Herzberg, 2003; Herzberg et al., 2010). La teoría de Herzberg sirvió para el desarrollo de la teoría X y Y elaborada por McGregor en su libro El lado humano de las organizaciones (Latham, 2007).

De otro lado, entre las teorías que describen la influencia social en la conducta del individuo se encuentran el condicionamiento clásico de Pavlov (Feldman, 2013), el conductismo (Skinner, 1981, 1987), la teoría de expectativas (Vroom, 1964) y la teoría de la equidad (Adams, 1963; Thierry, 2001).

La teoría conductista permite entender que los patrones observables de la conducta se encuentran condicionados por estímulos externos tanto en los animales como en los seres humanos. El determinismo que muestra Skinner en sus escritos tiene relación con los fenómenos ambientales (externos) y con las percepciones de esos fenómenos que ocurren en el organismo (interno), que en últimas generan respuestas o acciones diversas que pueden llegar a limitarse y regularse por medio de recompensas y sanciones (Delprato \& Midgley, 1992; Skinner, 1981, 1987).

Skinner se valió del método de aproximaciones sucesivas, que consiste en ir reforzando un comportamiento análogo o similar al que se desea reforzar a través de mecanismos de condicionamiento operatorio, esto es, refuerzos tanto negativos (castigos) como positivos (recompensas). Luego de que se ha logrado regular, se buscan nuevas formas de refuerzo tendientes a desarrollar conductas cercanas a las que quien investiga desea, tantas veces sea necesario, hasta lograr inducir al animal o al ser humano a mostrar comportamientos que, por voluntad propia, nunca hubiera desarrollado (Skinner, 1981, 1987).

Por su parte, Vroom (1964) argumenta que la conducta humana es el resultado de elecciones conscientes hechas por individuos entre distintos cursos de acción para obtener la mayor cantidad de gratificación o la disminución del dolor. Esta teoría supone que las personas se motivarán para realizar algo cuando consideran que el cumplimiento de la tarea los llevará al logro de un objetivo o meta deseada de segundo orden (ver Figura 2). De esa manera, la gente escoge conscientemente un 
doi: http://dx.doi.org/10.15359/ree.21-2.5

URL: http://www.una.ac.cr/educare

CORREO: educare@una.cr

curso de acción determinado como consecuencia de su deseo de maximizar su placer o minimizar su dolor, basándose en sus propias percepciones, actitudes y creencias (Mitchell \& Daniels, 2003).

Vroom (1964) argumenta la existencia de tres percepciones en el proceso de toma de decisiones para alcanzar un objetivo: expectativa, instrumentalidad y la valencia. La expectativa se refiere a la probabilidad de que la participación en un acto determinado o comportamiento guiará a un conjunto de resultados determinados, a estos se les llama resultados de primer orden. La expectativa es relacionada con las distintas percepciones de los sujetos empleados y diferentes niveles de confianza, en términos de lo que son capaces de hacer. En segundo lugar, la instrumentalidad representa la percepción individual de la fuerza y la naturaleza de la relación entre la consecución de los resultados de primer orden y el logro posterior de un segundo conjunto de resultados, llamado resultados de segundo orden. En otras palabras, se refiere a la evaluación subjetiva o percepción del personal acerca de si un mejor rendimiento en su trabajo en realidad logrará un aumento en el salario o un ascenso deseado (Mitchell \& Daniels, 2003).

La valencia es entendida por Vroom (1964) como el valor otorgado por una persona empleada a un determinado conjunto de resultados de segundo orden: vale decir, las orientaciones emocionales que poseen respecto de recompensas deseadas como el elogio de las jefaturas, el aumento de salario, seguridad en el trabajo, entre otros (ver Figura 2).

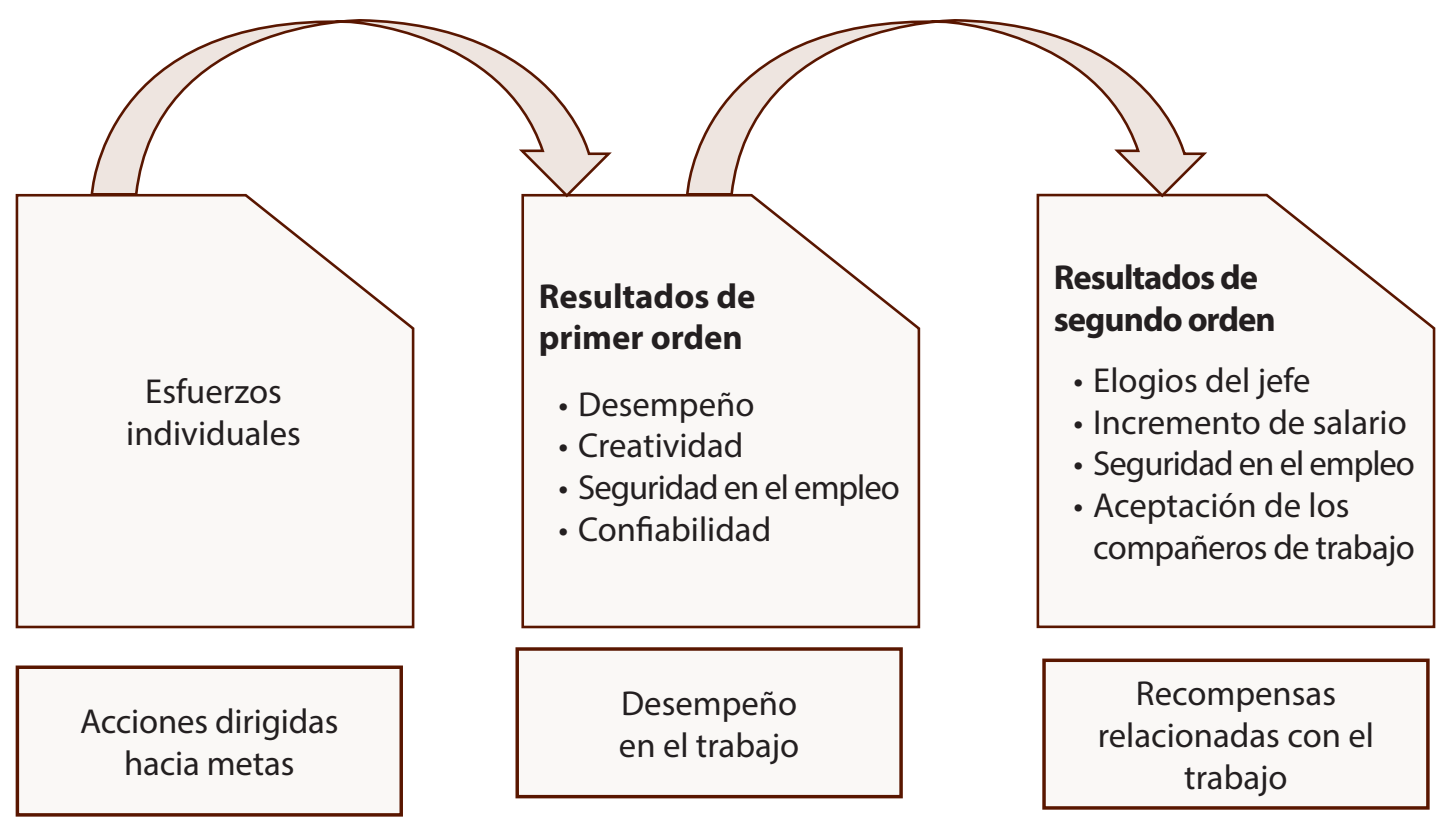

Figura 2: Elaboración propia a partir de Vroom (1964). 
doi: http://dx.doi.org/10.15359/ree.21-2.5

URL: http://www.una.ac.cr/educare

CORREO: educare@una.cr

Finalmente, la teoría de la equidad se ocupa de la percepción de los grupos trabajadores respecto de su participación dentro de una organización. Se define como un proceso de intercambio en el que ofrecen una serie de inputs, como su conocimiento, experiencia, esfuerzo, nivel de educación y, a cambio, reciben unos beneficios (salarios, promociones, reconocimiento). Estos beneficios pueden percibirse de dos maneras: tangibles, como el salario e intangibles, como las relaciones interpersonales (Mitchell \& Daniels, 2003).

La idea central de esta teoría es que los individuos se motivan cuando existe equidad en el proceso de intercambio en términos de los beneficios que reciben en comparación con el esfuerzo o empeño puesto en la tarea. En otras palabras, hay motivación cuando se percibe que los esfuerzos de un individuo se ven recompensados con los beneficios que recibe de una organización (Adams, 1963).

Las personas evalúan la equidad de este intercambio a través de un proceso social de comparación en el cual consideran la razón entre el desempeño percibido por sí mismas y los beneficios obtenidos (razón de equidad) en comparación con un tercer sujeto, que puede ser un compañero o compañera de trabajo o de los beneficios percibidos de un trabajo previo, al que llamaremos "referente de equidad". En ese sentido, se compara la razón de equidad con el referente de equidad y, si existe una aproximación entre ambos, se dice que es justo y por tal motivo un sujeto trabajador puede sentirse satisfecho (Donovan, 2002). En caso contrario, si una persona percibe una discrepancia entre estas dos ratios (es decir, si es mejor o peor pagado) se produce un estímulo adverso en proporción a la magnitud de la inequidad.

Adams (1963) intenta predecir si las personas estarán motivadas en situaciones en las cuales prevalece la justicia o la equidad. En recientes investigaciones se ha logrado demostrar que el desempeño de una persona empleada, en relación con su salario, crea información que influencia su percepción acerca de sí misma. Por ejemplo, aquellas personas que son mejor pagadas tienden a ver que su desempeño es mejor que el de otros individuos con salarios menores y, además, creen que poseen mayor control sobre los resultados de la organización que sus contrapartes (Thierry, 2001).

En la Tabla 2 se puede observar una breve descripción de cada teoría que se enfoca en estudiar la motivación extrínseca y sus principales exponentes. 
Tabla 2: Teorías de la motivación extrínseca

\begin{tabular}{|c|c|c|}
\hline Escuela & Principales exponentes & Breve descripción \\
\hline $\begin{array}{l}\text { Motivación en el } \\
\text { trabajo }\end{array}$ & (Herzberg, 2003) & $\begin{array}{l}\text { Las condiciones de trabajo no constituyen objetos motivantes, } \\
\text { son las motivaciones intrínsecas las que generan la mayor } \\
\text { satisfacción en el individuo. }\end{array}$ \\
\hline Teoría X y Y & (McGregor, 1971) & $\begin{array}{l}\text { Para el autor existen dos modelos de gerencia, cada uno con } \\
\text { una perspectiva acerca del individuo: Modelo X, la persona es } \\
\text { motivada primordialmente por incentivos económicos. Modelo } \\
\text { Y, el sujeto es motivado por cuenta propia, haciendo uso del } \\
\text { autocontrol. }\end{array}$ \\
\hline $\begin{array}{l}\text { Condicionamiento } \\
\text { operante }\end{array}$ & (Skinner, 1981, 1987) & $\begin{array}{l}\text { Aborda la conducta humana asumiendo que el comportamiento } \\
\text { es determinado por factores externos (es aprendido) y no por } \\
\text { medio de lo interno (reflexivo o no aprendido). Al crear situaciones } \\
\text { positivas (agradables y deseables) en donde se presentarán } \\
\text { comportamientos específicos, tendría como consecuencia una } \\
\text { mayor probabilidad de repetición de esas conductas en el futuro. }\end{array}$ \\
\hline $\begin{array}{l}\text { Teoría de las } \\
\text { expectativas }\end{array}$ & (Vroom, 1964) & $\begin{array}{l}\text { El desempeño y la motivación en el lugar de trabajo están } \\
\text { relacionados con las aspiraciones futuras del individuo, es decir, } \\
\text { con el alcance de metas de segundo orden como ascensos, } \\
\text { bonificaciones, entre otros. }\end{array}$ \\
\hline $\begin{array}{l}\text { Teorías acerca del } \\
\text { estado de ánimo, la } \\
\text { emoción y el afecto }\end{array}$ & $\begin{array}{l}\text { (George \& Brief, } \\
\text { 1992, 1996; Weiss \& } \\
\text { Cropanzano, 1996; Weiss, } \\
\text { Suckow \& Cropanzano, } \\
\text { 1999) }\end{array}$ & $\begin{array}{l}\text { Esta teoría busca la relación entre la satisfacción laboral del } \\
\text { individuo y su estado deánimoy sus emociones. La satisfacción } \\
\text { laboral puede ser entendida como un estado emocional } \\
\text { positivo o placentero, consecuencia de la evaluación del } \\
\text { trabajo o de las experiencias vividas en el ambiente laboral. }\end{array}$ \\
\hline Teoría de la equidad & $\begin{array}{l}\text { (Adams, 1963; Thierry, } \\
\text { 2001) }\end{array}$ & $\begin{array}{l}\text { Se ocupa de la percepción del personal respecto de su } \\
\text { participación dentro de una organización. Se define como un } \\
\text { proceso de intercambio en el que los individuos empleados } \\
\text { ofrecen una serie de inputs, como su conocimiento, } \\
\text { experiencia, esfuerzo, nivel de educación y, a cambio, reciben } \\
\text { unos beneficios (salarios, promociones, reconocimiento). }\end{array}$ \\
\hline
\end{tabular}

Nota: Elaboración propia a partir de Donovan (2002) y Mitchell \& Daniels (2003).

\section{Metodología}

Para el abordaje de esta investigación exploratoria, se recurrió a la construcción de una encuesta que evalúa la percepción del personal directivo de las instituciones de educación secundaria en cuatro áreas específicas: dotación física del colegio, motivación intrínseca docente, motivación extrínseca (sistemas de incentivos) y el impacto social de la institución. 
doi: http://dx.doi.org/10.15359/ree.21-2.5

URL: http://www.una.ac.cr/educare

CORREO: educare@una.cr

Para el primer conjunto de preguntas (dotación física) se escogieron una serie de factores higiénicos (Herzberg, 2003) para evaluar las percepciones del grupo entrevistado, con el objeto de recabar información necesaria para la comparación entre colegios públicos y privados en este tópico. Para medir sus percepciones acerca de la motivación intrínseca y extrínseca que muestran docentes en sus colegios, se formularon preguntas que responden a las teorías descritas en el marco teórico desarrollado. Por último, se utilizó una escala inspirada en la teoría de Maslow para determinar la percepción relacionada con el impacto social de la institución y, de esta forma, hallar elementos de comparación entre los colegios públicos y los privados.

Para el análisis de los datos se utilizó una escala de Likert cuyo rango inicia en (1) En total desacuerdo y culmina en (5) Totalmente de acuerdo (Likert, 1932).

La escala de Likert es una escala ordinal que permite medir la percepción o actitud de un individuo frente a un fenómeno estudiado en grados que van desde lo menos favorable (1) hasta la calificación más favorable (5). Esta escala utiliza afirmaciones que son calificadas por el individuo de la siguiente manera: Total desacuerdo (1), en desacuerdo (2), indiferente o neutro (3), de acuerdo (4), totalmente de acuerdo (5). Las respuestas posteriormente se tabulan y se promedian. Como toda escala ordinal, este procedimiento no permite medir la distancia de las percepciones entre dos individuos, en otras palabras, es difícil encontrar la distancia de un sujeto que ha contestado "de acuerdo", con la de otro que ha contestado "en desacuerdo".

El instrumento se aplicó en cincuenta y un colegios (51) del área metropolitana de Medellín, la cual comprende los municipios de Barbosa, Girardota, Bello, Medellín, Itagüí, Sabaneta, Estrella, Copacabana y Caldas en el departamento de Antioquia, Colombia. 18 de esos colegios son privados y 33 son públicos. La recolección de los datos se elaboró de forma directa en cada institución, a través de una encuesta a personal rector o coordinador académico. En promedio, existen 46,63 docentes por institución, y cada docente cumple con un promedio de 23,55 horas de clase directas. Para el manejo y análisis estadístico de los datos se utilizaron el software SPSS y Excel, y para calcular la confiabilidad de los datos se recurrió al coeficiente de Cronbach.

\section{Discusión y resultados}

\section{Resultados}

En la Tabla 3 se pueden observar los resultados del estudio exploratorio, se contrastan las respuestas del personal rector o coordinador académico de colegios públicos y colegios privados ubicados en la zona metropolitana de Medellín, involucrados en el estudio. 
Tabla 3: Resultados de la encuesta elaborada a personal rector o coordinador académico de las instituciones de educación secundaria en Medellín y su área metropolitana

\begin{tabular}{|c|c|c|c|c|}
\hline \multirow[b]{2}{*}{ Factor evaluado } & \multicolumn{2}{|c|}{ Colegio privado } & \multicolumn{2}{|c|}{ Colegio público } \\
\hline & $\bar{x}$ & $\sigma$ & $\bar{x}$ & $\sigma$ \\
\hline $\begin{array}{c}\text { Dotación de la institución } \\
\text { Dotación biblioteca }\end{array}$ & 4,1667 & 0,9852 & 3,2727 & 1,2317 \\
\hline Dotación aulas de clase & 4,2222 & 0,8782 & 3,5758 & 1,0009 \\
\hline Dotación laboratorios & 3,6111 & 1,2433 & 3,1515 & 1,0642 \\
\hline Zonas verdes para el disfrute & 2,8333 & 1,6539 & 2,7576 & 1,5619 \\
\hline Disponibilidad y estado de cafeterías & 3,5000 & 1,2485 & 3,3636 & 1,1407 \\
\hline Ayudas técnicas para las aulas & 4,2222 & 0,8782 & 3,1212 & 1,1112 \\
\hline Oficinas para docentes, & 4,0556 & 1,1100 & 3,1818 & 1,2363 \\
\hline Gimnasio o áreas deportivas & 3,6667 & 1,2834 & 2,4242 & 1,4149 \\
\hline $\begin{array}{l}\text { Motivación intrínseca } \\
\text { Compromiso de docentes con las actividades académicas } \\
\text { en el aula de clase }\end{array}$ & 4,1667 & 0,5145 & 4,2121 & 0,5453 \\
\hline Compromiso de docentes con las actividades pedagógicas & 3,9444 & 0,7254 & 4,0909 & 0,7230 \\
\hline $\begin{array}{l}\text { La dirección de la institución tiene en cuenta las } \\
\text { recomendaciones y sugerencias que efectúan docentes }\end{array}$ & 4,3333 & 4,0000 & 3,8485 & 0,8704 \\
\hline $\begin{array}{l}\text { El personal docente de su institución realiza sus actividades } \\
\text { por vocación }\end{array}$ & 4,0556 & 1,1100 & 3,8788 & 0,6963 \\
\hline $\begin{array}{l}\text { Motivación extrínseca } \\
\quad \text { Estabilidad laboral de docentes }\end{array}$ & 4,3889 & 0,6077 & 4,5455 & 0,6170 \\
\hline $\begin{array}{l}\text { Existen en la institución remuneraciones por fuera } \\
\text { del salario pactado }\end{array}$ & 2,3889 & 1,4608 & 1,7188 & 1,3255 \\
\hline La institución ofrece capacitaciones periódicas al personal docente & 3,5000 & 1,0432 & 3,1818 & 1,1580 \\
\hline El salario de docentes es suficiente para cubrir sus necesidades básicas & 3,8889 & 0,7584 & 2,8125 & 1,0298 \\
\hline $\begin{array}{l}\text { Indicador de impacto social } \\
\text { Es considerado su colegio de alto rendimiento, según logros } \\
\text { obtenidos en estudiantado egresado y en pruebas del Estado }\end{array}$ & 4,0000 & 1,0290 & 3,6667 & 0,8539 \\
\hline $\begin{array}{l}\text { El entorno social que rodea la institución es seguro para las } \\
\text { actividades de docencia }\end{array}$ & 4,1111 & 0,7584 & 3,8182 & 0,6826 \\
\hline Existe producción académica por parte de docentes & 3,4444 & 1,2935 & 2,8182 & 1,1580 \\
\hline $\begin{array}{l}\text { El entorno familiar y de apoyo es favorable para desarrollar las } \\
\text { actividades docentes }\end{array}$ & 4,2222 & 0,8782 & 3,2121 & 1,1112 \\
\hline
\end{tabular}

Nota: Los autores a partir de los datos obtenidos en la encuesta. 
doi: http://dx.doi.org/10.15359/ree.21-2.5

URL: http://www.una.ac.cr/educare

CORREO: educare@una.cr

De otro lado, se midió la percepción de favorabilidad acerca de los factores estudiados en la encuesta aplicada, de acuerdo con la Figura 3:

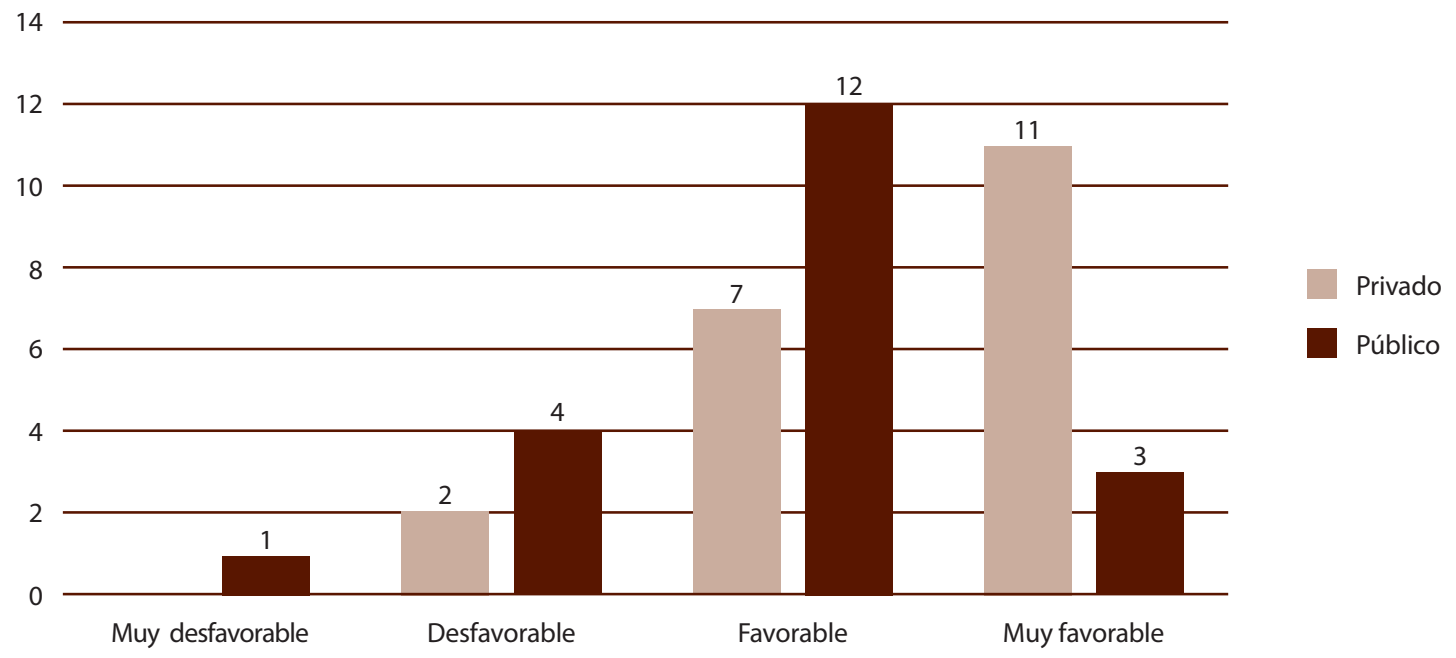

Figura 3: Sumatoria de factores para determinar la percepción de favorabilidad en instituciones de educación secundaria en la zona metropolitana de Medellín, 2015.

Nota: Elaboración propia a partir de los datos recolectados en la encuesta.

\section{Discusión}

Seobservan profundas diferencias entre las buenas calificaciones obtenidas por los colegios privados respecto de los públicos, especialmente en las áreas relacionadas a continuación:

- Ayudas y tecnología disponibles en el aula.

- Gimnasios y áreas para el deporte.

- Salarios del personal docente vinculado.

- El entorno familiar y de apoyo favorable al desarrollo de las actividades académicas.

- Producción intelectual.

Los datos muestran que en los colegios privados hay un mayor índice de favorabilidad tanto por los espacios y dotación de la institución, como por los indicadores de impacto social cuya medición es, en conjunto, mayor a la obtenida en los colegios públicos. 
En contraste, la medición de la percepción acerca de la motivación docente, tanto intrínseca como extrínseca, no arroja diferencias sustanciales entre los colegios públicos y privados, salvo en el factor salarial, donde la calificación supera en un punto en los privados por encima de los públicos.

Lo anterior coincide con el estudio previo elaborado por los autores, en el cual el factor salarial fue uno de los peores calificados (Franco-López et al., 2015); en esa medición se obtuvo que 46 de 283 docentes vinculados a colegios públicos respondieron favorablemente a la pregunta, es decir, el $16 \%$ de docentes manifestaba insatisfacción con su salario. Esto permite inferir que el tema salarial es uno de los principales tópicos que requieren de atención en la institución escolar y que puede ser objeto de investigaciones futuras.

\section{Validez de los datos}

La confiabilidad de los datos se midió a través de un coeficiente de Conbrach a través de la fórmula 1:

$$
x=\frac{K}{K-1}\left[1-\frac{\sum_{i}^{k}=1 s_{i}^{2}}{S_{i}^{2}}\right]
$$

Donde:

$S_{i}^{2}$ es la varianza del ítem $\mathrm{i}$,

$S_{i}^{2}$ es la varianza de los valores totales observados y

$\mathrm{K}$ es el número de preguntas o ítems.

Como resultado de la operación, se obtuvo un coeficiente alfa de 0,809. De esta manera, se considera que los datos poseen una consistencia adecuada.

\section{Conclusiones}

De acuerdo con los datos recolectados, según las percepciones de personal directivo de los colegios estudiados, existe una mayor favorabilidad para el personal docente en los colegios privados que en los públicos del área metropolitana de Medellín, según lo mostrado en la mayoría de tópicos evaluados. Los resultados muestran que la percepción de personal rector y director académico acerca de la motivación docente es similar en ambos tipos de instituciones; sin embargo, el factor salarial (considerado un motivador extrínseco) es el principal elemento diferenciador, dada la baja calificación obtenida en los colegios públicos respecto de los privados. 
doi: http://dx.doi.org/10.15359/ree.21-2.5

URL: http://www.una.ac.cr/educare

CORREO: educare@una.cr

Así mismo, se observa que existe una baja calificación en ambos tipos de instituciones, tanto públicas como privadas, en la pregunta que evalúa la existencia de remuneraciones extra o sistemas de incentivos para docentes, esto confirmaría la tesis de Deci (1972), según la cual los incentivos se consideran accesorios y minarían la motivación hacia la obtención de metas de sus docentes. En contraste, las preguntas que miden las percepciones acerca del compromiso docente con las actividades académicas y pedagógicas en el aula de clase obtuvieron calificaciones altas.

Por último, los hallazgos de este estudio exploratorio, que mide la percepción de personal directivo de cincuenta y un colegios, acerca de la motivación de su profesorado, concuerda y complementa los hallazgos elaborados en un estudio previo, donde se encontró que los principales factores de motivación docente se localizan en factores intrínsecos, especialmente en uno de ellos, la vocación. Ambos estudios concuerdan que el personal docente siente agrado tanto por la profesión como por su trabajo; sin embargo, considera inequitativas las diferencias salariales entre colegios públicos y privados. Este es un elemento extrínseco de la motivación que deberá ser revisado por los entes gubernamentales para mejorar las condiciones salariales de los grupos docentes, en aras de la calidad educativa, se incentiva en especial a docentes contratados en el decreto 1278 de 2002.

El tema no se agotaría exclusivamente en los salarios, hay otras formas alternativas para analizar, los grupos docentes deben mejorar sus ingresos a través de sistemas de incentivos por producción académica, apoyos en tiempos y dinero (becas) para el desarrollo de estudios de alta formación (doctorado y postdoctorado), así como en otro tipo de beneficios (factores extrínsecos) que permitan mejorar las condiciones laborales y la calidad de la enseñanza del área analizada.

\section{Referencias}

Achtziger, A., Gollwitzer, P. M., \& Sheeran, P. (2008). Implementation intentions and shielding goal striving from unwanted thoughts and feelings. Personality and Social Psychology Bulletin, 34(3), 381-393. doi: https://doi.org/10.1177/0146167207311201

Adams, J. S. (1963). Towards an understanding of inequity. The Journal of Abnormal and Social Psychology, 67(5), 422-436. doi: https://doi.org/10.1037/h0040968

Alderfer, C. P. (1972). Existence, relatedness, and growth. Human needs in organizational settings. New York, NY: Free Press.

Amabile, T. M. (1997). Motivating creativity in organizations: On doing what you love and loving what you do. California Management Review, 40(1), 39-58. doi: https://doi. org/10.2307/41165921

Araque, J., \& Rivera, N. (2005). Psicología organizacional e industrial. Bogotá: Ecoe. 
Bandura, A. (1988). Self-regulation of motivation and action through goal systems. En V. Hamilton, G. Bower, \& N. Frijda (Eds.), Cognitive perspectives on emotion and motivation (pp. 31-61). Norwell, MA: Kluwer Academic Publishers. doi: https://doi.org/10.1007/978-94-009-2792-6 2

Bandura, A. (1991). Social cognitive theory of self-regulation. Organizational Behavior and Human Decision Processes, 50(2), 248-287. doi: https://doi.org/10.1016/0749-5978(91)90022-L

Bennis, W. G. (1966). Changing organizations: Essays on the development and evolution of human organization. New York: McGraw-Hill.

Campion, M. A., \& Lord, R. G. (1982). A control systems conceptualization of the goal-setting and changing process. Organizational Behavior and Human Performance, 30(2), 265-287. doi: https://doi.org/10.1016/0030-5073(82)90221-5

Carbonero, M. Á., Martín-Antón, L. J., Román, J. M., \& Reoyo, N. (2010). Efecto de un programa de entrenamiento al profesorado en la motivación, clima de aula y estrategias de aprendizaje de su alumnado. Revista Iberoamericana de Psicología y Salud, 1(2), 117-138. Recuperado de http://www.redalyc.org/articulo.oa?id=245116406001

Carver, C. S., \& Scheier, M. F. (1981). Attention and self-regulation. A control-theory approach to human behavior. New York, NY: Springer Verlag. doi: https://doi.org/10.1007/978-1-4612$\underline{5887-2}$

Carver, C. S., \& Scheier, M. F. (1990). Origins and functions of positive and negative affect: A controlprocess view.Psychological Review, 97(1), 19-35. doi: https://doi.org/10.1037/0033-295X.97.1.19

Davis, K., \& Newstron, J. (2003). Comportamiento humano en el trabajo (10ª ed.). México: McGrawHill Interamericana.

Day, C., Sammons, P., Stobart, G., Kington, A. y Gu, Q. (2007). Teachers matter: Connecting lives, work and effectiveness. New York: McGraw-Hill.

Deci, E. L. (1972). The effects of contingent and noncontingent rewards and controls on intrinsic motivation. Organizational Behavior and Human Performance, 8(2), 217-229. doi: https:// doi.org/10.1016/0030-5073(72)90047-5

Deci, E. L. (1980). The psychology of self-determination. Toronto: Lexington Books.

Delprato, D.J., \& Midgley, B. D. (1992). Some fundamentals of B. F. Skinner's behaviorism. American Psychologist, 47(11), 1507-1520. doi: https://doi.org/10.1037/0003-066X.47.11.1507

Donovan, J. (2002). Work motivation. En N. Anderson, D. S. Ones, H. K. Sinangil, \& C. Viswesvaran (Eds.), Handbook ofindustrial, work \& organizational psychology (Vol. 2, pp.53-76).Thousand Oaks, California: SAGE. 
doi: http://dx.doi.org/10.15359/ree.21-2.5

URL: http://www.una.ac.cr/educare

CORREO: educare@una.cr

Emo, W. (2015). Teachers' motivations for initiating innovations. Journal of Educational Change, 16(2), 171-195. doi: https://doi.org/10.1007/s10833-015-9243-7

Feldman, R. (2013). Essentials of understanding psychology (11 a ed.). New York: McGraw-Hill.

Franco-López, J. A., López-Arellano, H., \& Vélez-Salazar, M. (2015). La influencia de la motivación docente en el proceso de aprendizaje. En G. Ramírez, J. A. Rosas, O. Lozano, M. D. Gil, M. T. Magallón, A. Alburquerque ... C. J. Núñez (Eds.), El análisis organizacional en México y América Latina. Retos y perspectivas a 20 años de estudios (Tomo I, pp. 2201-2240). México D.F.: Universidad Autónoma de Itztapalapa. Retrieved from http://remineo.org/images/ documentos/memorias/ciao/xiiiciao/aomal.pdf

Gagné, M., \& Deci, E. L. (2005). Self-determination theory and work motivation. Journal of Organizational Behavior, 26(4), 331-362. doi: https://doi.org/10.1002/job.322

Gagné, M., \& Deci, E. L. (2014). The history of self-determination theory in psychology and management. En M. Gagné (Ed.), The oxford handbook of work engagement, motivation, and self-determination theory (pp. 1-9). Oxford: Oxford University Press. doi: https://doi. org/10.1093/oxfordhb/9780199794911.013.006

George, J. M., \& Brief, A. P. (1992). Feeling good-doing good: a conceptual analysis of the mood at work-organizational spontaneity relationship. Psychological Bulletin, 112(2), 310-329. doi: https://doi.org/10.1037/0033-2909.112.2.310

George, J. M., \& Brief, A. P. (1996). Motivational agendas in the workplace: The effects of feelings on focus of attention and work motivation. Research in Organizational Behavior, 18, 75-109.

Gollwitzer, P. M. (1999). Implementation intentions: Strong effects of simple plans. American Psychologist, 54(7), 493-503. doi: https://doi.org/10.1037/0003-066X.54.7.493

Gollwitzer, P. M., \& Sheeran, P. (2006). Implementation intentions and goal achievements: A meta-analysis of its effects and processes. In M. P. Zanna (Ed.), Advances in experimental social psychology (Vol. 38, pp. 69-119). New York, NY: Academic Press. doi: https://doi. org/10.1016/s0065-2601(06)38002-1

González, M. (2003). Claves para favorecer la motivación de los profesores ante los retos educativos actuales. Estudios Sobre Educación, 5, 61-83. Recuperado de http://dadun.unav. edu/bitstream/10171/8471/1/Estudios\%20Ed.pdf

Herrera, D., \& Matos, L. (2009). Desarrollo del concepto de motivación y su representación en distintas aproximaciones teóricas. En D. Herrera, Teorías contemporáneas de la motivación. Una perspectiva aplicada (pp. 17-36). Lima: Fondo Editorial de la Pontificia Universidad Católica del Perú. 
Herzberg, F. (2003). Una vez más: ¿Cómo motiva usted a sus empleados? Harvard Business Review, 81(1), 67-76.

Herzberg, F., Mausner, B., \& Bloch, B. (2010). The motivation to work (12 ed.). New Jersey: Transaction Publisher.

Kirkpatrick, S. A., \& Locke, E. A. (1991). Leadership: do traits matter? The Executive, 5(2), 48-60. doi: https://doi.org/10.5465/AME.1991.4274679

Latham, G. P. (2007). Work motivation: History, theory, research, and practice. Thousand Oaks: SAGE.

Lauretti, P., Villalobos, E., \& González, J. (2007). Programa de motivación en el aula: Una experiencia para el desarrollo del docente en ejercicio. Revista Mexicana de Orientación Educativa, 4(10), 15-23. Recuperado de http://pepsic.bvsalud.org/pdf/remo/v4n10/a04.pdf

Likert, R. (1932). A technique for the measturement of attittudes. Archives of Psychology, 22(140), 1-55.

Locke, E. A., \& Latham, G. P. (2002). Building a practically useful theory of goal setting and task motivation: A 35-year odyssey. American Psychologist, 57(9), 705-717. doi: https://doi. org/10.1037/0003-066X.57.9.705

Locke, E. A., \& Latham, G. P. (2013). Goal Setting Theory, 1990. En E. A. Locke, \& G. P. Latham (Eds.), New developments in goal setting and task performance (pp. 3-15). New York, NY: Routledge.

Locke, E. A., Shaw, K. N., Saari, L. M., \& Latham, G. P. (1981). Goal setting and task performance: 19691980. Psychological Bulletin, 90(1), 125-152. doi: https://doi.org/10.1037/0033-2909.90.1.125

Maslow, A. H. (1991). Motivación y personalidad. Madrid: Ediciones Díaz de Santos.

McClelland, D. C. (1989). Estudio de la motivación humana. España: Narcea.

McGregor, D. (1971). El aspecto humano de las empresas. México: McGraw-Hill.

Ministerio de Educación. (14 de septiembre de 1979). Decreto 2277 de 1979. Recuperado de http://www.mineducacion.gov.co/1621/articles-103879 archivo pdf.pdf

Ministerio de Educación. (19 de junio de 2002). Decreto 1278. Recuperado de http://www. mineducacion.gov.co/1621/articles-86102 archivo pdf.pdf

Mitchell, T. R., \& Daniels, D. (2003). Motivation. En I. Weiner, W. Borman, D. Ilgen, \& R. Klimoski (Eds.), Handbook of psychology (Vol. 12, pp. 225-254). New Jersey: John Wiley \& Sons, Inc. doi: https://doi.org/10.1002/0471264385.wei1210

Moretti, M. M., \& Higgins, E. T. (1999). Internal representations of others in self-regulation: A new look at a classic issue. Social Cognition, 17(2), 186-208. doi: https://doi.org/10.1521/ $\underline{\text { soco.1999.17.2.186 }}$ 
doi: http://dx.doi.org/10.15359/ree.21-2.5

URL: http://www.una.ac.cr/educare

CORREO: educare@una.cr

Murillo, G., Zapata, Á., Martínez, J., Á, Ávila, H., Salas, J., \& López, H. (2007). Teorías clásicas de la organización y el management. Cali: Ecoe.

Navarro, E. (2008). Aportación al estudio de la satisfacción laboral de los profesionales técnicos del sector de la construcción: Una aplicación cualitativa en la Comunidad Valenciana (Tesis doctoral). Universidad Politécnica de Valencia, España. Recuperado de https://riunet.upv. es/bitstream/handle/10251/2189/tesisUPV2784.pdf

Panadero, E., \& Alonso-Tapia, J. (2014). Teorías de autorregulación educativa: Una comparación y reflexión teórica. Psicología Educativa, 20(1), 11-22. doi: https://doi.org/10.1016/j. pse.2014.05.002

Ringelhan, S., Wollersheim, J., Welpe, I. M., Fiedler, M., \& Spörrle, M. (2013). Work motivation and job satisfaction as antecedents of research performance: Investigation of different mediation models. En A. Dilger, H. Dyckhoff, \& G. Fandel (Eds.), Performance Management im Hochschulbereich (Vol. 3, pp. 7-38). Wiesbaden: Springer Fachmedien Wiesbaden. doi: https://doi.org/10.1007/978-3-658-03348-4 2

Rodríguez, S., Núñez, J. C., Valle, A., Blas, R., \& Rosario, P. (2009). Auto-eficacia docente, motivación del profesor y estrategias de enseñanza. Escritos de Psicología, 3(1), 1-7. Retrieved from http://www.redalyc.org/articulo.oa?id=271020403001

Romero, G.-A. (2009). La motivación del profesor: Un gran recurso educativo. Innovación y experiencias educativas, 20, 1-9. Recuperado de http://www.csi-csif.es/andalucia/modules/ mod ense/revista/pdf/Numero 20/GUSTAVO-ADOLFO ROMERO BAREA02.pdf

Skinner, B. F. (1981). Ciencia y conducta humana (Una psicología científica) (5 ed.). Barcelona: Editorial Fontanella.

Skinner, B. F. (1987). Sobre el conductismo. Barcelona: Planeta de Agostini.

Thierry, H. (2001). The reflection theory on compensation. En M. Erez, U. Kleinbeck, \& H. Thierry (Eds.), Work motivation in the context of a globalizing economy (pp. 149-166). New Jersey: Lawrence Erlbaum.

Torres, J. (2006). La desmotivación del profesorado. Madrid: Morata.

Vroom, V. H. (1964). Work and motivation. Oxford, UK: Wiley.

Weinberg, R., Gould, D., \& Jackson, A. (1979). Expectations and performance: An empirical test of Bandura's self-efficacy theory. Journal of Sport Psychology, 1(4), 320-331. doi: http://dx.doi. org/10.1123/jsp.1.4.320 
doi: http://dx.doi.org/10.15359/ree.21-2.5

URL: http://www.una.ac.cr/educare

Weiss, H. M., \& Cropanzano, R. (1996). Affective Events Theory: A theoretical discussion of the structure, causes and consequences of affective experiences at work. En B. M. Staw \& L. L. Cummings (Eds.), Research in organizational behavior: An annual series of analytical essays and critical reviews (Vol. 18, pp. 1-74). US: JAI Press.

Weiss, H. M., Suckow, K., \& Cropanzano, R. (1999). Effects of justice conditions on discrete emotions. Journal of Applied Psychology, 84(5), 786-794. doi: https://doi.org/10.1037/0021$\underline{9010.84 .5 .786}$

Wood, R., \& Bandura, A. (1989). Impact of conceptions of ability on self-regulatory mechanisms and complex decision making. Journal of Personality and Social Psychology, 56(3), 407-415. doi: https://doi.org/10.1037/0022-3514.56.3.407 